\title{
Deconvolution of non-Gaussian linear processes with vanishing spectral values
}

\author{
(non-minimum phase/cumulant spectra/filter/noise)
}

\section{Keh-SHIN LiI* AND MURRay RosenblatT ${ }^{\dagger}$ \\ *Department of Statistics, University of California, Riverside, CA 92521; and tDepartment of Mathematics, University of California at San Diego,} La Jolla, CA 92093

Contributed by Murray Rosenblatt, September 9, 1985

\begin{abstract}
We consider the problem of estimating the filter generating a non-Gaussian linear process and the deconvolution of that process when the spectral density of the process has zeros. Without using a minimum phase assumption we show that often if there are only finitely many zeros there are procedures to effect such an estimation and deconvolution.
\end{abstract}

Linear processes $\mathbf{X}_{t}$ are generated by an independent identically distributed input sequence $\eta_{t}$ convolved with a linear filter $\alpha_{t}$

$$
\mathbf{x}_{t}=\sum_{j} \alpha_{j} \eta_{t-j}
$$

This model is of considerable interest in geoexploration (14). Given observations on $X_{t}$ only, a main interest is to estimate the filter coefficients $\alpha_{t}$ and the input sequence $\eta_{t}$ by deconvolving $\mathbf{X}_{t}$.

Let $\alpha(z)=\Sigma \alpha_{j} z^{j}$ be the $z$-transform of the filter $\alpha_{t}$. Then the spectral density of $\left\{X_{r}\right\}$ is $f(\lambda)=\sigma_{\eta}^{2}\left|\alpha\left(e^{-i \lambda}\right)\right|^{2} / 2 \pi$, which is completely determined by the second-order properties of $\left\{\mathbf{X}_{t}\right\}$ and does not contain the phase information of $\alpha(z)$. If $\eta$ has a Gaussian distribution then the process $\left\{\mathbf{X}_{t}\right\}$ is completely determined by second-order properties and one can only determine $\alpha(z)$ up to $|\alpha(z)|$. There is the indeterminancy of the phase of $\alpha(z)$. Often this is resolved by making the ad hoc assumption that $\alpha(z)$ is minimum phase. If $\alpha(z)$ is polynomial this means the roots are outside the unit disc.

Often, $\eta_{t}$ appears to have a non-Gaussian distribution and then one need not assume $\alpha(z)$ minimum phase. By using information provided by the higher-order (greater than 2) cumulants, the phase of $\alpha(z)$ can be estimated. Results using higher-order cumulant spectral techniques have been given by Rosenblatt (5) and by Lii and Rosenblatt $(3,6)$. A main result is summarized below.

LEMMA. Consider a non-Gaussian linear process $\mathbf{X}_{\mathrm{t}}$ given in Eq. 1 with $\eta_{t}$ having all their moments finite. Let $\Sigma\left|j \alpha_{j}\right|<$ $\infty$ and assume $\alpha\left(\mathrm{e}^{-\mathrm{i} \lambda}\right) \neq 0$ for all $\lambda$. The transfer function $\alpha\left(\mathrm{e}^{-\mathrm{i} \lambda}\right)$ can then be identified in terms of observations only on $\left\{\mathbf{X}_{\mathrm{t}}\right\}$ up to an indeterminate integer $\mathrm{a}$ in a factor $\mathrm{e}^{\mathrm{ia \lambda}}$ that corresponds to a shift of index of $\mathbf{X}_{t}$ and an indeterminate sign of $\alpha(1)=\Sigma \alpha_{\mathrm{k}}$. For this result it is sufficient to have some finite moment of order $\mathrm{k}>2$ with cumulant $\gamma_{\mathrm{k}} \neq 0$.

The basic idea is to obtain the modulus of $\alpha\left(e^{-i \lambda}\right)$ using the spectral density $f(\lambda)$ of $\left\{\mathbf{X}_{t}\right\}$ [since $\left.\left|\alpha\left(e^{-i \lambda}\right)\right|=|2 \pi f(\lambda)|^{1 / 2}\right]$ assuming $E \eta_{t} \equiv 0$ and $E \eta_{t}^{2} \equiv 1$. There are a number of consistent estimates of $f(\lambda)$ discussed by Priestley (7). The phase $h(\lambda)=\arg \left\{\alpha\left(e^{-i \lambda}\right) \alpha(1) /|\alpha(1)|\right\}$ of $\alpha\left(e^{-i \lambda}\right)$ is obtained

The publication costs of this article were defrayed in part by page charge payment. This article must therefore be hereby marked "advertisement" in accordance with 18 U.S.C. $\$ 1734$ solely to indicate this fact. by making use of the relation

$$
\begin{aligned}
h\left(\lambda_{1}\right) & +\cdots+h\left(\lambda_{k-1}\right)-h\left(\lambda_{1}+\cdots+\lambda_{k-1}\right) \\
& =\arg \left[\left\{\frac{\alpha(1)}{|\alpha(1)|}\right\}^{k} \gamma_{k}^{-1} b_{k}\left(\lambda_{1}, \ldots, \lambda_{k-1}\right)\right],
\end{aligned}
$$

which is derived from the $k$ th-order cumulant spectral density of the process $\left\{\mathbf{X}_{t}\right\}$

$$
\begin{aligned}
& b_{k}\left(\lambda_{1}, \ldots, \lambda_{k-1}\right) \\
& =\frac{\gamma_{k}}{(2 \pi)^{k-1}} \alpha\left(e^{-i \lambda_{1}}\right) \cdots \alpha\left(e^{-i \lambda_{k-1}}\right) \alpha\left(e^{i\left(\lambda_{1}+\cdots+\lambda_{k-1}\right)}\right) .
\end{aligned}
$$

Given an estimate $\hat{b}_{k}$ of $b_{k}$ one can obtain an estimate $\hat{h}(\lambda)$ of $h(\lambda) . \hat{\alpha}\left(e^{-i \lambda}\right)=\left|\hat{\alpha}\left(e^{-i \lambda}\right)\right| e^{i \hat{h}(\lambda)}$ is an estimate of $\alpha\left(e^{-i \lambda}\right)$. One can deconvolve $\mathbf{X}_{t}$ and obtain estimates of $b_{k}$ by using

$$
\hat{\eta}_{t}=\hat{\alpha}^{-1} * \mathbf{X}_{t} .
$$

\section{THE RESULTS}

In many contexts the spectral density $f(\lambda)$ does have zeros and it is of some interest to see whether one can carry out deconvolution and in the case of this model recover the original non-Gaussian white noise sequence $\eta_{n}$. We have already remarked that, under the assumption of the lemma, Condition $A$ is valid.

Condition $A$. The function $\alpha\left(e^{-i \lambda}\right)$ can be consistently estimated up to a factor $e^{i a \lambda}$ with a an integer and the sign \pm 1 .

In the results we mention, the important details of how to estimate $f(\lambda)$ and $\alpha\left(e^{-i \lambda}\right)$ will not be dealt with in any detail here. Some of the aspects of estimation of $\alpha\left(e^{-i \lambda}\right)$ can be found in the papers by Lii and Rosenblatt $(3,6)$.

One should note that Condition $A$ will be satisfied under the following assumption concerning $\alpha\left(e^{-i \lambda}\right)$.

PROPOSITION. If $\alpha\left(\mathrm{e}^{-\mathrm{i} \lambda}\right)$ is the restriction to the unit circle of a function $\alpha(\mathrm{z}) \neq 0$ analytic in an annulus about the unit circle, Condition A is satisfied. Such a function can have at most a finite number of zeros on $|\mathrm{z}|=1$.

Under this assumption the procedure suggested via equations 2 and 3 of the paper for the estimation of $\alpha\left(e^{-i \lambda}\right)$ will still be asymptotically valid (see additional remark at end of paper).

THEOREM 1. Under Condition A the process $\mathbf{X}_{n}$ can be deconvolved so as to obtain the process $\eta_{\mathrm{n}}$ up to sign and a time shift by making use of the filter corresponding to

$$
\left\{\mathrm{f}_{\mathrm{n}}(\lambda)+\mathrm{a}_{\mathrm{n}}\right\}^{-1 / 2} \exp \left\{-\mathrm{i} \arg \alpha_{\mathrm{n}}\left(\mathrm{e}^{-\mathrm{i} \lambda}\right)\right\},
$$

where $\mathrm{f}_{\mathrm{n}}(\lambda)(\geqslant 0), \alpha_{\mathrm{n}}\left(\mathrm{e}^{\mathrm{i} \lambda}\right)$ are appropriate consistent estimates of $\mathrm{f}(\lambda), \alpha\left(\mathrm{e}^{-\mathrm{i} \lambda}\right)$ based on a sample size $\mathrm{n}$ and $\mathrm{a}_{\mathrm{n}}$ an appropriate sequence of positive numbers with limit zero. 
We shall also consider the case in which the process $x_{n}$ has added to it Gaussian white noise $\xi_{t}$ with small variance $a>0$. We thus have the observed process

$$
\begin{aligned}
\xi_{n}= & x_{n}+\xi_{n}=\int_{-\pi}^{\pi} e^{i n \lambda} \alpha\left(e^{-i \lambda}\right) d z_{\eta}(\lambda) \\
& +\int_{-\pi}^{\pi} e^{i n \lambda} d z_{\xi}(\lambda)
\end{aligned}
$$

with $z_{\eta}(\lambda), z_{\xi}(\lambda)$ random spectral processes corresponding to the processes $\eta$ and $\xi$.

THEOREM 2. Under Condition $A$ and $\Sigma\left|j \mathrm{j}_{\mathrm{j}}\right|<\infty$, the process $\mathrm{X}_{\mathrm{n}}$ can be deconvolved so as to obtain the process $\eta_{\mathrm{n}}$ up to sign and a time shift by applying the filter corresponding to Eq. 5 to the process $\xi_{\mathrm{n}}$ and letting $\mathrm{n} \rightarrow \infty$.
Details, particularly those concerned with estimation and derivation of results, will be provided later.

This research was partially supported by Office of Naval Research Contract N00014-81-K0003 and National Science Foundation Grant DMS83-12106.

1. Wiggins, R. (1978) Geoexploration 17, 21-35.

2. Jurkevics, A. \& Wiggins, R. (1984) Geophysics 49, 2109-2116.

3. Lii, K. S. \& Rosenblatt, M. (1982) Ann. Stat. 10, 1195-1208.

4. Matsuoka, T. \& Ulrych, T. J. (1984) Proc. IEEE 72, $1403-$ 1411.

5. Rosenblatt, M. (1980) J. Appl. Probab. 17, 265-270.

6. Lii, K. S. \& Rosenblatt, M. (1985) Multivariate Analysis (Elsevier, New York), Vol. 6, pp. 395-410.

7. Priestley, M. B. (1981) Spectral Analysis and Time Series (Academic, New York). 